\title{
Induction of Human Glioma Tumor in Sprague-Dawley Rats with Intact Immune System
}

\author{
Zahra ABDI I,2, Hossien ESKANDARY',3, Seyed Noureddin NEMATOLLAHI-MAHANI2,4 \\ ${ }^{1}$ Department of Anatomy, Afzalipour School of Medicine, Kerman University of Medical Sciences, Kerman, Iran \\ ${ }^{2}$ Neuroscience Research Center, Institute of Neuropharmacology, Kerman University of Medical Sciences, Kerman, Iran \\ ${ }^{3}$ Afzal Research Institute (NGO), Kerman, Iran \\ ${ }^{4}$ Department of Anatomy, School of Medicine, Zanjan University of Medical Sciences, Zanjan, Iran
}

\section{ABSTRACT}

AIM: Glioblastoma (GBM) is an aggressive brain tumor in humans. The median survival rate of patients is one year after the diagnosis. So, development of an animal model is necessary for the advances in the research treatment of GBM. The aim of this study was to investigate the capability of human glioma cells in inducing glioma tumors in rats with intact immune system.

MATERIAL and METHODS: U87 cells were implanted in the frontal lobe of rats without suppressing the immune system. We used magnetic resonance imaging (MRI), Hematoxylin-Eosin (H\&E) and Immunohistochemical (IHC) staining to assess characteristics of tumor.

RESULTS: At the $10^{\text {th }}$ and $14^{\text {th }}$ days of tumor inoculation, MRI images contained the tumor areas in the brain. All tumor-bearing rats developed tumors. The rats retained the morphology and histological characteristics of human glioma. Animals mimic GBM characteristics, such as mitotic activity, invasion, neovascularization, necrosis and pseudopalisading cells. IHC staining revealed tumor growth and progression in the tumor-bearing rats.

CONCLUSION: This model is a standard system for studying the tumor phenotype, genotype, and for evaluating the efficacy of anti-cancer agents. It is a reliable, simple, inexpensive, and easily reproducible model, which may be a way for pre-clinical studies.

KEYWORDS: U87 cell, Animal model, Rat, Glioblastoma, Immunohistochemistry

\section{INTRODUCTION}

$\mathrm{G}$ lioblastoma (GBM) is a common and aggressive type of brain tumor in humans. Despite improvement in therapy techniques, patients have a short life expectancy (12-15 months) and GBM is an incurable disease (7,32). The inefficacy of current treatment methods stimulates the researchers to seek novel therapeutic agents and strategies. So, development of laboratory and animal model is a substantial step for the advances in the treatment of GBM $(19,23)$.

An animal model is an important tool for the understanding of complex phenomena involved in glioma generation; and it provides the possibility to investigate the histological and genetic characteristics of neoplasms (11). Commonly applied models that generate brain neoplasms in animals include:

a) Chemical mutagen-induced models (36),

b) Genetic modification-induced models (2),

c) Xenograft induced models $(20,30)$.

These models have led to better understanding of the mechanisms related to tumor progression. However, these models are not comprehensive, and each of the models has its own issues $(18,31)$. The major drawbacks of chemically induced brain tumors include their histological characteristics 
that are different from the tumor, and the tumor latency period is long and variable $(4,36)$. Genetic modification-induced models are dependable and powerful in investigation of tumor genetic aspects. The main disadvantage is the difficulty of production, high price, and also the cost of the breeding and maintenance (22).

Xenograft is the most common brain tumor-induced model. It has histopathological characteristics that are similar to human glioblastoma $(11,20,30)$. The hosts of the xenograft model are often nude mice or rats with a suppressed immune system. The main limitation of this model is the absence of an intact immune system $(22,24)$. In normal conditions, the component of the immune system including antibody-antigen interaction, T-cells, different T-cell sub-populations, and natural killer cells play an important role $(16,24)$. Recent advances in immunology have led to a better understanding of the interactions between the tumors and immune system. Numerous evidences described major aspects of T-cells, and T-cell subpopulations in the progression or rejection of the tumors. Moreover, there are crosstalks between tumor cells and immune system cells, which facilitates growth and invasion of the tumor (34). Thus, the existence of the active immune system is essential in order to exact study of the tumor initiation and growth process. However, an appropriate experimental model for the analysis of growth and tumor cells-immune system interaction in human glioma tumors has not been described yet. Therefore the ability of human glioma cells to form tumor mass in normal immune-system rats would provide a suitable model in which immune system interactions in the progression and treatment of glioma could be investigated. In the present study, our aim was the induction of human glioma tumor in male SpragueDawley rats without suppressing their immune system.

\section{MATERIAL and METHODS}

This study was approved by the Ethical Commission of the Kerman University of Medical Sciences with Approval number $\mathrm{EC} / \mathrm{KNRC} / 86 / 23$.

\section{U87 Cells}

The U87 cell line was purchased from the Pasteur Institute (Tehran, Iran). The cells were cultured in Dulbecco's modified Eagle's medium (DMEM) with $10 \%$ fetal bovine serum (FBS) and $1 \%$ Penicillin/streptomycin in the humidified atmosphere at $37^{\circ} \mathrm{C}$ with $5 \% \mathrm{CO}_{2}$. The cells were labeled with $\mathrm{PKH}_{26}$ dye according to the manufacture's protocol. Briefly, the cells were trypsinized, centrifuged, counted, and about $10^{7}$ viable cells were exposed to $\mathrm{PKH}_{26}$ as recommended by the manufacturer.

\section{Animals}

Fourteen male Sprague-Dawley rats weighing 220-250 g were used for the experiments. The rats were housed in the animal room, 12/12 hours day/night cycle with free access to rodents chow and drinking water. These rats were randomly divided into 2 groups of 7 rats: phosphate-buffered solution (PBS group), and tumor (tumor cells in PBS).

\section{Implantation of U87-PKH ${ }_{26}$ Cells}

Animals were anesthetized intraperitoneally with using a mixture of ketamine/xylazine. Then, the head of the animals were shaved and the animals were fixed in a stereotaxic frame. The scalp was incised in the midline of the skull. Three holes were drilled in the right half of the skull. The coordinates of the holes were as follow:

A) lateral $=2 \mathrm{~mm}$, anterior $=1 \mathrm{~mm}$,

B) lateral $=3 \mathrm{~mm}$, anterior $=1 \mathrm{~mm}$,

C) lateral $=2 \mathrm{~mm}$, anterior $=2 \mathrm{~mm}$ ) of the Bregma point.

The Hamilton syringe was fixed on the arm of the stereotaxic frame. The needle was inserted to the depth of $3-4 \mathrm{~mm}$ of the brain and $10 \mu \mathrm{l}$ of cell suspension ( $10^{6}$ cells in PBS) was injected slowly $(1 \mu \mathrm{l} / 1 \mathrm{~min})$ in each of the holes. After 3 minutes the needle was withdrawn gently and the holes were covered with bone wax and the incision was sutured with 4-0 suture. The same procedure was carried out for PBS group, except the cells were excluded.

\section{Magnetic Resonance Imaging (MRI)}

MRI was performed on the animals of the tumor group 7,10 and 14 days after tumor inoculation and also an animal in the PBS group. The animals were anesthetized by ketamine/xylazine. The rats were positioned head-up in the knee coil of 1.5-Tesla unite (AVAN TO Siemens imaging system, Germany), and T1-weighted spin-echo images (repetition time $=\mathrm{TR}=400 \mathrm{~ms}$, echo time $=\mathrm{TE}=19 \mathrm{~ms}$, slice thickness $=2 \mathrm{~mm}$ ), T2-weighted spin-echo images (repetition time $=\mathrm{TR}=2000 \mathrm{~ms}$, echo time $=\mathrm{TE}=72 \mathrm{~ms}$, slice thickness $=2 \mathrm{~mm}$ ), were obtained. 14 days after implantation of tumor, the rats were anesthetized, and were perfused with $4 \%$ paraformaldehyde. The brains were post-fixed by $10 \%$ buffered formalin for 24 hours and were immersed in $30 \%$ sucrose in PBS for 48 hours.

\section{Staining}

For H\&E and IHC Staining, paraffin-embedded $3 \mu \mathrm{m}$ tissue sections were mounted on slides. Following deparaffinization \& rehydration, numbers of sections were incubated with primary antibodies at the following concentrations: Monoclonal mouse Anti-Human Ki-67 (Dako, Carpinteria, CA, USA), monoclonal mouse anti-human Nestin (Santa Cruz Biotechnology), mouse monoclonal antibody glial fibrillary acidic protein (GFAP)(Santa Cruz Biotechnology, USA); PECAM-1 monoclonal mouse Anti-Rat CD-31 (Bio-Rad); diluted in PBS, 1:100. Slides were incubated with biotinylated secondary antibodies against rat/ rabbit followed by incubation with the streptavidin/biotinylated peroxidase complex (Dako, Peroxidase/DAB, Rabbit/Mouse). Sections were counterstained with hematoxylin. Some of the sections were stained with H\&E stain.

To detect $\mathrm{PKH}^{+}{ }_{26}$ cells in the brain tissue, the brains were immediately frozen in Tissue-Tek OCT embedding gels. Cryostat-generated $7 \mu \mathrm{m}$ sections were mounted onto slides and examined under a fluorescence microscope (IX 71, Olympus, Japan) equipped with a digital camera. The proportion of Ki-67, Nestin, GFAP, and CD-31 positive cells was calculated as the percentage of the stained cells in 200 cells. 


\section{Statistical Analysis}

Distribution of data was analyzed by the Kolmogorov-Smirnov test and paired Sampel T-test was carried out with IBM SPSS Statistics version 21 . A p value $<0.05$ was considered to detect a statistically significant difference. Data are expressed as mean \pm SEM.

\section{- RESULTS}

\section{MRI}

MRI was performed in both groups to assess tumor development and cerebral injuries. MRI was performed at 7 , 10 and 14 Days. On MRI T2-W and T1-W images of tumor group, which were performed on the seventh day from tumor implantation, no signs of cerebral injuries and tumor growth were seen (Figure 1A). On T2-W images of 10 days from tumor inoculation, there was a hyperintense spot in the white matter of the right frontal lobe without obvious damage. On coronal T1-W images, the spot was iso- to hypointense in the tumor group (Figure 1B). Formation of solid tumor masses occurred rapidly, after 10 days of U87 cells inoculation; and 14 days of tumor inoculation, the T1-W and T2-W images of
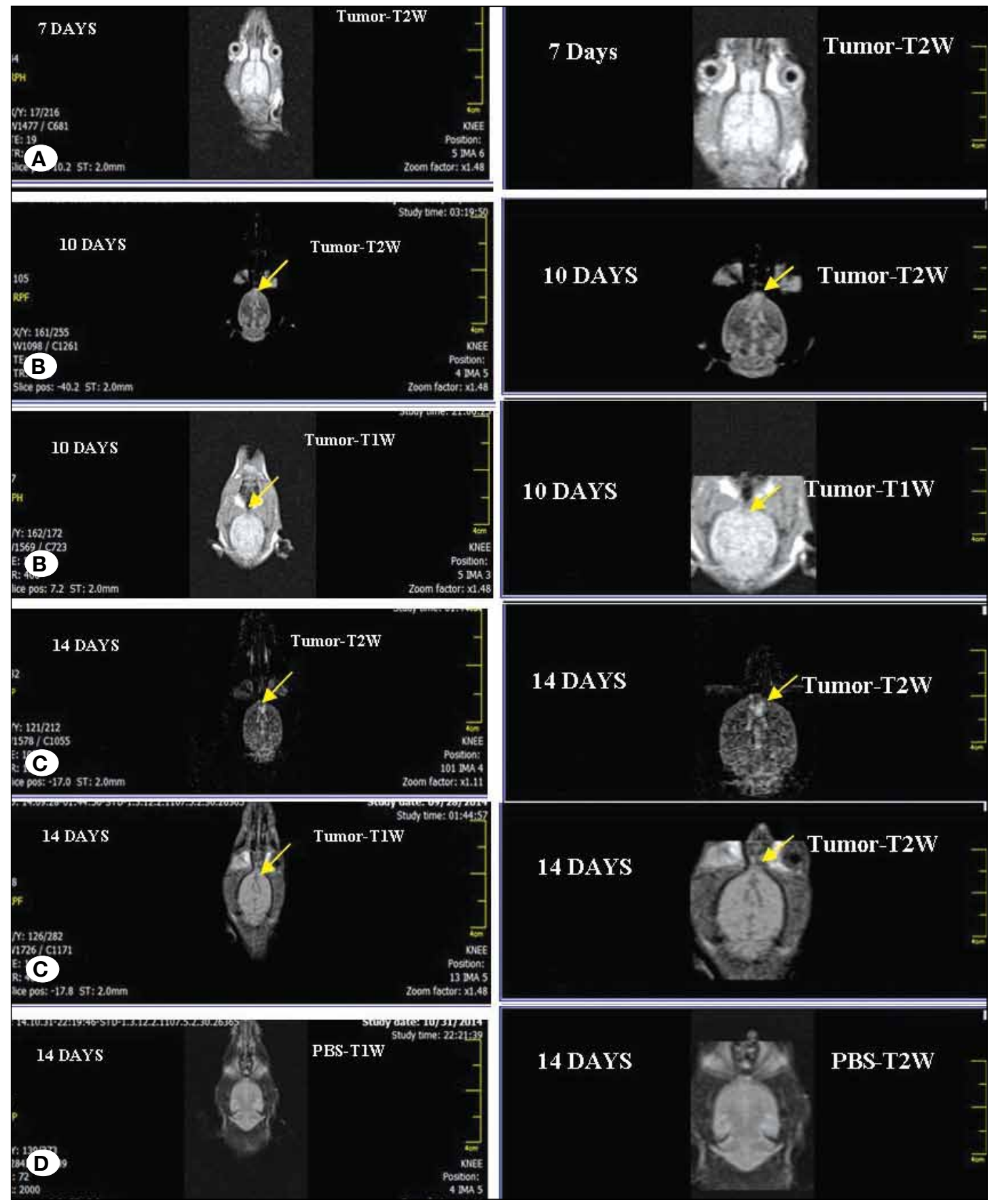

Figure 1: Magnetic resonance imaging of both groups. 
the tumor-bearing animals contained three spots with clear contrast in right frontal lobe and on coronal T2-W, three hyperintense spots were seen. On T1-W, spots were observed iso- to hypointense with increased contrast (Figure 1C). MRI $\mathrm{T} 1-\mathrm{W}$ and $\mathrm{T} 2-\mathrm{W}$ images of the control group did not show signs of cerebral injuries such as cyst formation, edema, and hemorrhage (Figure 1D).

\section{Histopathology}

In this study, frozen serial sections were examined with a fluorescence microscope for the detection of tumors. Three tumor areas were clearly seen. To show that U87 cells remained as whole cells in the brain of rats, frozen sections were counterstained with Hoechst (a nuclear specific dye). Dual $\mathrm{PKH}^{+}{ }_{26}$ and $\mathrm{Hoechst}^{+}$cells were seen in the brain of the rats (Figures $2 \mathrm{~A}-\mathrm{C}$ ).

H\&E staining in U87 inoculated animals revealed that glioma masses stained strongly with hematoxylin compared with the normal parenchyma (Figure 3A). The presence of neoplastic characteristics such as mitotic activity, migration of tumor cells, neovascularization, necrotic clusters along with pseudopalisading cells, and hemorrhage was observed in the tumor group (Figures 3B-F). Tumor cells were seen with normal and bizarre mitotic figures (Figure 3B). Most of the tumor cells were observed normal with heterochromatic, round, and oval nuclei (Figure 3B). Interestingly, U87 cells have migrated into the normal brain parenchyma and they were found in the white matter and normal parenchyma as well (Figure 3B). Invading cells were characterized by a heterochromatic nucleus (Figure $3 \mathrm{~B})$. Migration of tumor cells in the perivascular space was observed (Figure 3C). Small necrotic foci were found only in four rats. Necrotic foci were surrounded by tumor cells (Figure 3D). In two of the rats, a necrotic center with pseudopalisading was observed (Figure 3D). Infiltration of inflammatory cells was seen adjacent to the tumor (Figure 3E). The findings of the tumor group depicted that numerous neovasculariza-

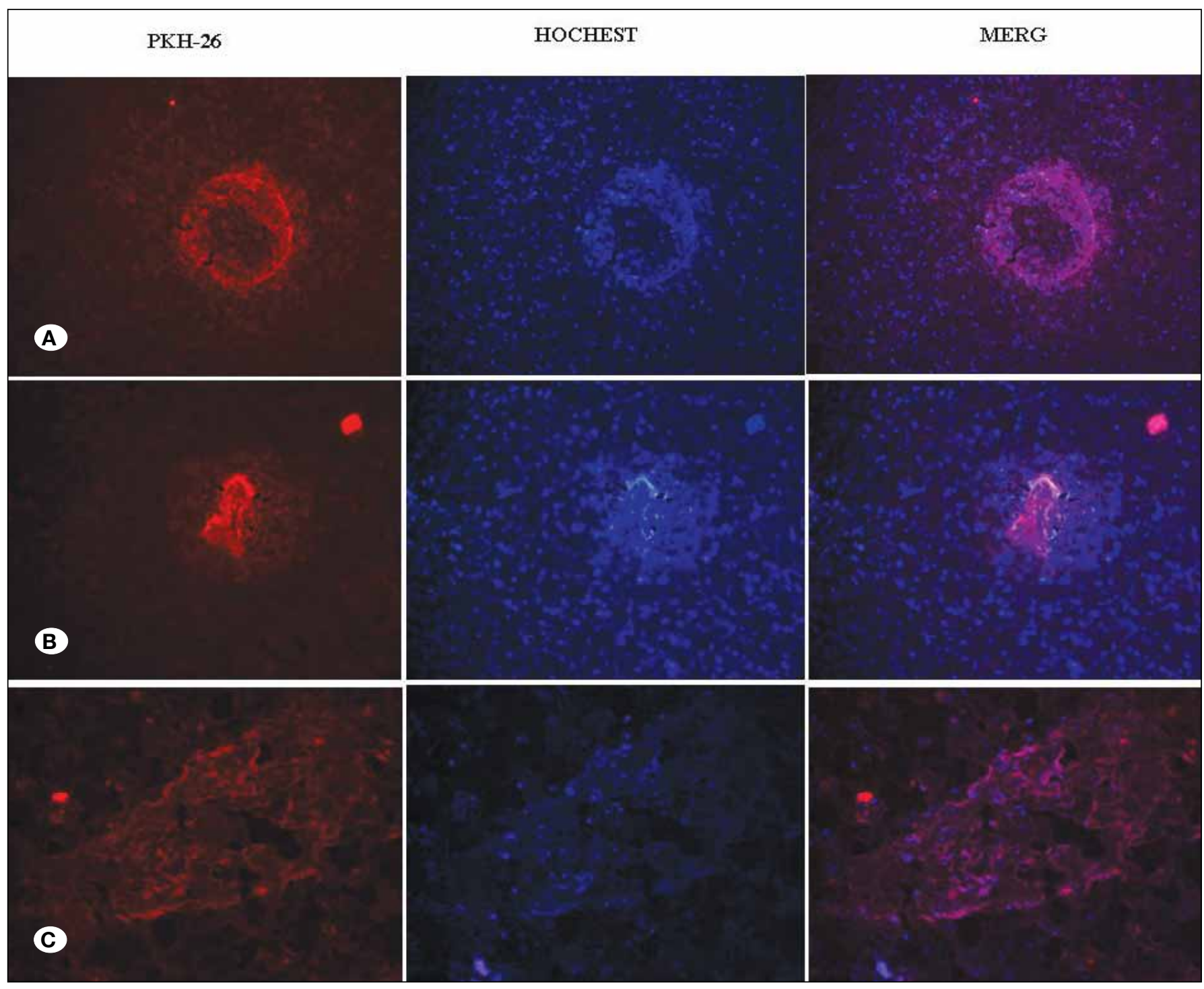

Figure 2: Frozen sections of tumoral tissues are shown at three sites of tumor implantation $(\times 200)(\mathbf{A}-\mathbf{C})$. 
tion areas were detected in all animals (Figure 3E). Moreover, hemorrhage was seen in three of the rats but it was not very intense (Figure 3F).

In the PBS group, infiltration of inflammatory cells, small blood vessels, and dead blood cells were observed (Figure 3G). Inflammatory cells and some dead blood cells were seen in the areas of injection (Figure $3 \mathrm{H}$ ).

Moreover, for neovascularization determination, $10 \mathrm{H \& E}$ sections were investigated randomly and new vessels were counted at a magnification of $\times 200$. In the tumor group, the neovascular proliferations occurred usually near the periphery of tumor mass or intratumoral areas. Our findings suggested that the rate of angiogenesis was increased in tumor-bearing rats, and a significant difference was found between the PBS and tumor groups for angiogenesis. In the tumor group, the rate of neovascularization was two times higher in comparison with the control group (Figures 3C,E and Figure 4A).

\section{Immunohistology}

The findings of our IHC analysis showed Ki-67 expression was vastly different between the control and tumor groups.
The proportion of $\mathrm{Ki}-67$ positive cells in the tumor group was significantly higher than the control group $(p<0.01$, Figure 4B). In comparison with the control group, Ki-67 was strongly expressed in glioma-bearing rats. Increase of the $\mathrm{Ki}-67$ positive cells revealed an increase in mitotic activity in the glioma masses of glioma-bearing rats (Figures $5 A-D$ ). Moreover, the numbers of Ki-67 positive cells increased in the glioma masses and surrounding area of the glioma; that their numbers decreased distance from the tumor masses (Figures $5 \mathrm{C}, \mathrm{D})$.

Our findings suggested that the protein level of Nestin in the tumor group was markedly upregulated in comparison with the control group. The rate of Nestin positive cells was $9.75 \%$ in the tumor group; which is significantly higher than the control groups (Figure 4B and Figures 5E-G). Moreover, the number of Nestin positive tumor cells was scattered around main tumor masses and their number decreased by increasing the distance of tumor areas (Figures 5F,G). As expected, expression rate was very low in the PBS group (Figure 5E).

IHC staining of GFAP detected a strong expression at the tumor periphery in tumor-bearing animals. Interestingly,

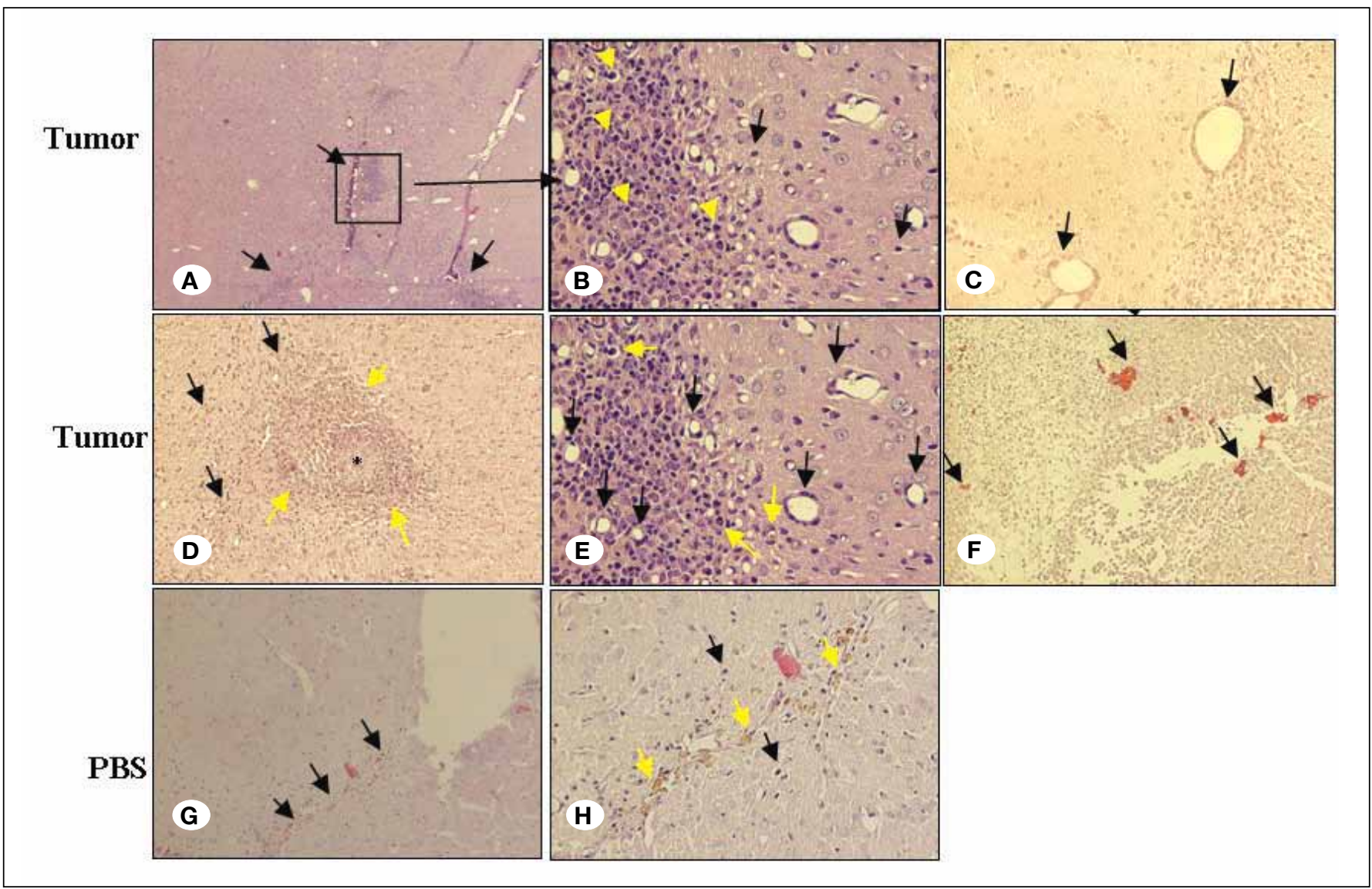

Figure 3: H\&E sections. A) Three sites of tumor implantation (×100). B) Tumor cells (yellow arrow) and migration of tumor cells into the normal parenchyma (black arrow) $(\times 320)$. C) Migration of tumor cells in perivascular space $(\times 200)$. D) Migration of tumor cells to the distance of tumor masses (black arrow) and Necrotic center (black star) showing pseudopalisading (yellow arrow) (×200). E) Infiltration of immune system cells (yellow arrow) and Neovascularization $(\times 320)$. F) Hemorrhage $(\times 200)$. G) Injection sites of PBS $(\times 100)$. H) Inflammatory cells (black arrow) and dead blood cells (yellow arrow) $(\times 200)$. 
GFAP was scantly expressed in tumor masses and highly expressed in borders and around the tumor areas. The rate of GFAP expression depicted a significant difference with the control group (Figure $4 \mathrm{~B}$ and Figures $5 \mathrm{H}, \mathrm{l})$. The results of $\mathrm{IHC}$ staining with endothelial-specific CD31 showed that gliomabearing rats had higher expression than rats in the PBS group. Moreover, the positive CD-31 cells were also detected in the main tumor mass, suggesting the development of new blood vessels (Figure 4B and Figures $5 \mathrm{~J}, \mathrm{~K}$ ). The overall number of CD-31 positive cells incresed dramatically in the glioma infected region; the tissues of the tumor group exhibited more than 5\% CD31 immunostained cells. It was also detectable in the control group, and a statistical difference between the groups was observed.

\section{- DISCUSSION}

Despite many animal models that have been developed for brain cancer research, unfortunately there is no simple, available, and comprehensive animal model for brain tumor studies $(16,17)$. In the present study, we focused on inducing the main characteristics of GBM in Sprague-Dawley rats without suppressing the immune system. This is the first report of implantation of U87 (human cell line) in the brain of immunocompetent rats that leads to the development of the tumor. In this study, gliomas were induced by injection of human tumoral cells (U87) into the frontal lobe of SpragueDawley rat hosts.

We also investigated the histopathological features of glioma in rats. Analysis of histological slides of the brain revealed prominent histological features of gliomas such as pleomorphism, nuclear atypia, mitotic activity, and necrosis (23). According to previous reports $(4,5,28)$, we expected tumor rejection in the animals due to different origin of cells and animals. However, we did not observe any regression of tumor after two weeks, and the tumorigenicity percentage of U87 cancer cells using the intracranial inoculation method was $100 \%$. There may be several reasons for this:

1) Previous reports of tumor regression were related to subcutaneously or intraperitoneally implanted tumor (ectopic cancer model) and the induction of tumor was not in the brain (Orthotopic) $(3,5,25,28)$. Often, the ectopic cancer model is reproved for not representing tumors in practice, because the microenvironment plays a vital role in the development or rejection of tumor cells (10). The brain is an immunological privileged site and neutrophil infiltration is lower in the brain compared to other tissues. Moreover, blood-brain barrier (BBB) penetration occurs only after cerebral injuries such as trauma or ischemia. Therefore, most tumors that have been developed in the brain do not evoke a strong immune response $(14,31)$.

2) In spite of the fact that U87 cells are antigenic, they can be non-immunogenic for rats (27). Hence, U87 cells led to either a weak immunological response or nonimmunological response in the brain of rats or the tumoral cells may not be able to express a high level of antigen for the rejection of the glioma. Similar results have been reported for U87 cells in immunosuppressed rats (35).
3) The third reason relates to the period of follow-up of the tumor. In the present study, the duration of follow-up was 2 weeks. It is possible that the immune system did not have enough time for tumor elimination in this time-frame. As a consequence, this time-frame was appropriate for tumor growth, without any sign of infectious risks associated with tumor suppression. So, the most important aspect of the present model is the ability of U87 cells to grow in rats with intact immune systems. Currently, most brain tumor models use animals with non-intact immune systems. However, these models provide important scientific information, but the role of an intact immune system has been ignored. The interaction of a normal immune system in the initiation, growth, development and the treatment of glioma is critical (34). Thus, this rat model of brain tumor may be used to study the role of the intact immune system in the progression of the tumor. Moreover, a better understanding of the intact immune system role in tumor development could certainly shed new insight on the development and production of anticancer agents.

In addition, we know that an animal model to study brain tumors should exactly reproduce the various natural features of the disorder. Here we demonstrate a simple, new model of brain cancer in the rat because rats are closely related to humans from the aspect of anatomy, physiology, and genetics, and rats represent an excellent model to study various brain tumors. It therefore represents an appropriate animal model for studying therapeutic techniques and agents of glioma. Thus, this model provides a convenient, useful, and reliable model for investigating brain tumors in this time-frame.

In this study, MRI and histological evaluation of the glioma showed a progressive extension of the glioma 15 days after inoculation of U87 cells. However, in the experimental MRI studies on rodents, most researchers use an animal-special MRI system with 3-17.6 Tesla (6,29). We have monitored tumor growth using a 1.5 Tesla MRI system at 7,10 , and 14 days from tumor-implantation. The tumor masses were observed at 10 and 14 days, whereas they were not detectable at 7 days. This is likely due to the the capacity of the 1.5T MRI system. Vince et al. (37) reported results similar to our data with the 1.5 T MRI system.

In the present model, analysis of histological slides of rats revealed a similar histophatological characteristic of human glioblastoma such as polymorphism, mitotic activity, invasion, necrosis, hemorrhage, and neoangiogensis $(1,23)$. Also, the histological analysis indicated that the fast growth of the glioma tumor was accompanied by migration and invasion of tumor cells to normal tissue. We observed migration of tumor cells into normal brain tissue and perivascular space. Moreover, the number of tumor cells decreased with remote of the tumor masses that our findings were similar with the other reports (8). Whereas, Miura et al. (26) described a low rate of U87 cell infiltration to the adjacent brain parenchyma in athymic rats. There are reports that show that the migration of tumor cells is associated with the ability to proliferate of tumoral cells. In addition, Our Ki-67 data also indicated that $\mathrm{Ki}-67$ staining increased in intensity throughout glioma masses 


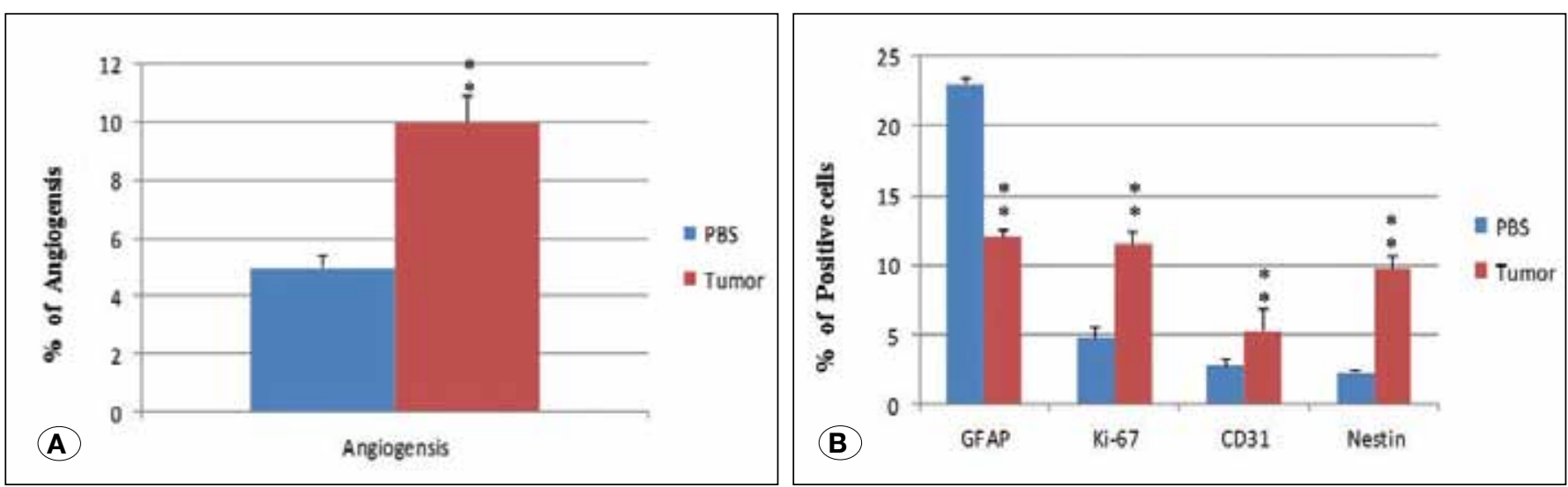

Figure 4: A) Angiogenesis analysis between groups. B) Immunohistology analysis of Nestin, CD-31, Ki-67 and GFAP in rats of both groups. Data are expressed as mean \pm SEM. ${ }^{*}, p<0.05,{ }^{* *}, p<0.01$.

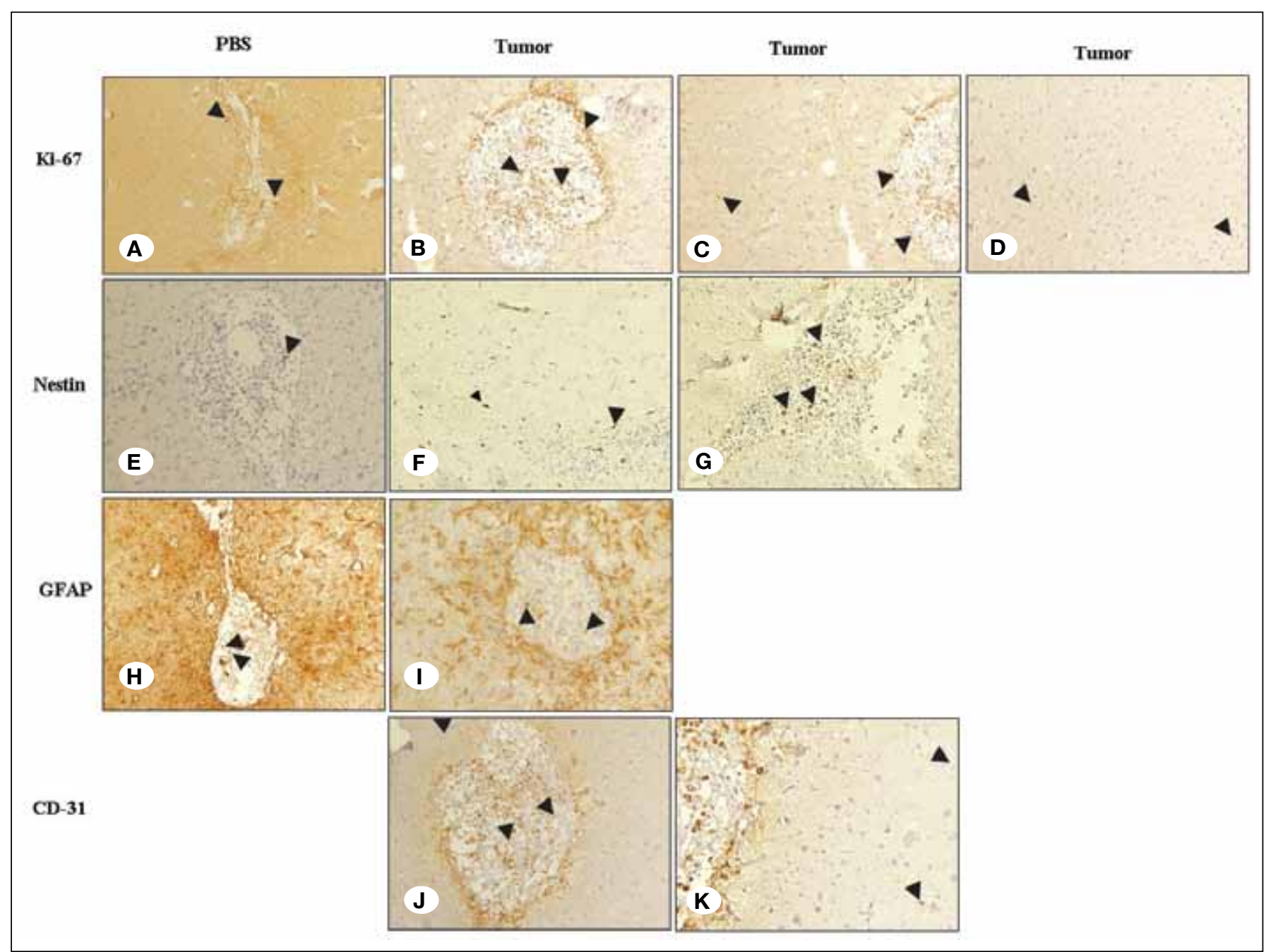

Figure 5: Immunostaining. A) Immunostaining of Ki-67; a few Ki-67 positive cell nuclei were detected in the injection area ( $\times 200)$. B) Ki-67 positive tumor cell in the tumor area $(\times 320)$. C) around the tumor area $(\times 400)$. D) far from the tumor area $(\times 400)$. E) Nestin $(\times 200)$. F-G) Nestin positive tumor cell in the tumor area and around the tumor area ( $\times 320)$. H) GFAP positive cells ( $\times 200)$. I) GFAP positive tumor cells in the tumor tissues $(\times 200)$. J) CD-31 positive cells in tumor area $(\times 320)$. K) positive cells near to main tumor masses $(\times 320)$. 
with a most prominent appearance in the edges of the glioma. Ki-67 shows the cells that get in synthesis plus G1 phases and we know that increased levels of $\mathrm{Ki}-67$ are indicative of the development of glioma; thus the proliferation and invasion of glioma cells were closely related to each other. The high expression of $\mathrm{Ki}-67$ could contribute to fast progression and aggressiveness in rats (21).

Another prominent feature of glioma is the microvascular proliferation that CD31 is one of the best markers for the measurement of vascularization. Numerous studies demonstrated the importance of angiogenesis in glioma growth (33). There are several reports about glioma cell invasion to the perivascular space. Reports demonstrated that the procedure of invasion is related to the angiogenesis and the proliferation of tumor cells $(13,38)$. In addition, hypoxia and necrotic centers stimulate the process of angiogenesis (15). Our data suggested the migration of U87 cells to the perivascular space and formation of novel microvascular structures. In our study, a well-known feature of the tumor group was microvascular formation that led to tumor cell proliferation and development of tumor masses. In addition, the CD31 results also indicated the formation of novel vascular structures around and in side the tumor, which was in accordance with the other reports (33). This model mimics both migration and neoangiogenesis as well as in human glioma. These observations, together with the prominent histological features of glioma, in rats suggest that this orthotopic model is an efficient animal model for studying glioma as well as an easy and simple implantation method for rapid tumorigenicity, and a simple monitoring method for tumor masses. Moreover, this model showed necrotic centers, pseudopalisading, and the presence of neoangiogenesis in side and around the tumor masses, that are prominent histological features of human glioma.

In this study, we observed an increased expression of GFAP in the borders of the tumor. Most papers indicated an increase of GFAP expression in the edge of tumor as well $(9,39)$. Recent reports suggested that increasing the number of Nestin positive cells in brain tumor accelerated the development of tumors and the expression rate of Nestin had been used as a marker to determine the prognosis in GBM patients (12). In our model, IHC staining indicated that Nestin had been expressed in the tumor masses periphery and mainly in the invasive tumor cells. Our IHC results confirmed previous reports. Therefore, the present model is reproducible and mimics glioma characteristics as observed in humans; and these findings indicate that the present rat model of brain cancer is amenable to study the pathological and genetic features of glioma and to measure the anti-cancer effects of various therapeutic agents such as chemotherapy, radiotherapy and anti-angiogenesis, in the treatment of glioma.

With these information, further investigations are needed to address the applicability of this model. Future studies comparing the properties of this model with human GBM and other animal models, and testing the drugs that have previously been tested in patients or other immune therapeutic modalities and chemotherapeutic modalities will be of great interest for the application of this model.

\section{CONCLUSION}

This study describes a rat model for potential use in the investigation of events related to glioma. Because of its simplicity, availability and the rapidity of tumor progression after inoculation of U87 cells, this model allows for the study of features of glioma; and it may be adopted in testing various anti-cancer drugs and agents. This novel model can serve as a valuable model in understanding the histology and biology of glioma and the present model may also be used for treatment methods such as chemotherapy and radiation. In addition, this model provides an opportunity for investigation of hosttumor interaction in the brain.

\section{- REFERENCES}

1. Adamson C, Kanu OO, Mehta Al, Di C, Lin N, Mattox AK, Bigner DD: Glioblastoma multiforme: A review of where we have been and where we are going. Expert Opin Investig Drugs 18: 1061-1083, 2009

2. Aguzzi A, Brandner S, Isenmann S, Steinbach JP, Sure U: Transgenic and gene disruption techniques in the study of neurocarcinogenesis. Glia 15: 348-364, 1995

3. Baldwin R: Immunity to methylcholanthrene-induced tumours in inbred rats following atrophy and regression of the implanted tumours. Br J Cancer 9: 652, 1955

4. Barth RF: Rat brain tumor models in experimental neurooncology: The 9L, C6, T9, F98, RG2 (D74), RT-2 and CNS-1 gliomas. J Neurooncol 36: 91-102, 1998

5. Beauchesne P, Bertrand S, Revel R, Pialat J, Brunon J, Mornex F, Doré J: Development of an intracerebral glioma model in whole body irradiated hairless rats. Anticancer Res 20: 703706, 1999

6. Beck B, Plant D, Grant S, Thelwall P, Silver X, Mareci T, Benveniste H, Smith M, Collins C, Crozier S: Progress in high field MRI at the University of Florida. MAGMA 13: 152-157, 2002

7. Black PM: Brain tumors. New Engl J Med 324: 1555-1564, 1991.

8. Candolfi M, Curtin JF, Nichols WS, Muhammad AG, King GD, Pluhar GE, McNiel EA, Ohlfest JR, Freese AB, Moore PF: Intracranial glioblastoma models in preclinical neurooncology: Neuropathological characterization and tumor progression. J Neurooncol 85: 133-148, 2007

9. Chekhonin V, Baklaushev V, Yusubalieva G, Pavlov K, Ukhova $O$, Gurina O: Modeling and immunohistochemical analysis of C6 glioma in vivo. Bull Exp Biol Med 143: 501-509, 2007

10. Cui ZY, Ahn JS, Lee JY, Kim WS, Lim HY, Jeon HJ, Suh SW, Kim JH, Kong WH, Kang JM: Mouse orthotopic lung cancer model induced by PC14PE6. Cancer Res Treat 38: 234-239, 2006

11. Dai C, Holland EC: Glioma models. Biochim Biophys Acta 1551: M19-M27, 2001

12. Ehrmann J, Kolář Z, Mokrý J: Nestin as a diagnostic and prognostic marker: Immunohistochemical analysis of its expression in different tumours. J Clin Pathol 58: 222-223, 2005 
13. Gasparini G, Harris AL: Clinical importance of the determination of tumor angiogenesis in breast carcinoma: Much more than a new prognostic tool. J Clin Oncol 13: 765-782, 1995

14. Greene HS: The transplantation of tumors to the brains of heterologous species. Cancer Res 11: 529-534, 1951

15. Hardee ME, Zagzag D: Mechanisms of glioma-associated neovascularization. Am J Pathol 181: 1126-1141, 2012

16. Holland EC: Brain tumor animal models: Importance and progress. Curr Opin Oncol 13: 143-147, 2001

17. Holland EC: Gliomagenesis: Genetic alterations and mouse models. Nat Rev Genet 2: 120-129, 2001

18. Huszthy PC, Daphu I, Niclou SP, Stieber D, Nigro JM, Sakariassen PØ, Miletic H, Thorsen F, Bjerkvig R: In vivo models of primary brain tumors: pitfalls and perspectives. Neuro-oncology 14: 979-993, 2012

19. Kanu OO, Mehta A, Di C, Lin N, Bortoff K, Bigner DD, Yan $\mathrm{H}$, Adamson DC: Glioblastoma multiforme: A review of therapeutic targets. Expert Opin Ther Targets 13: 701-718, 2009

20. Khachigian L, Field S, Crouch R, Chesterman C: Plateletderived growth factor A-chain synthetic peptide inhibits human glioma xenograft proliferation in nude mice. Anticancer Res 15: 337-341, 1994

21. Khoshyomn S, Penar PL, Rossi J, Wells A, Abramson DL, Bhushan A: Inhibition of phospholipase $\mathrm{C}-\mathrm{\gamma} 1$ activation blocks glioma cell motility and invasion of fetal rat brain aggregates. Neurosurgery 44: 568-577, 1999

22. Kondo S, Kondo Y, Li G, Silverman RH, Cowell JK: Targeted therapy of human malignant glioma in a mouse model by $2-5 \mathrm{~A}$ antisense directed against telomerase RNA. Oncogene 16: 3323-3330, 1998

23. Lacroix M, Abi-Said D, Fourney DR, Gokaslan ZL, Shi W, DeMonte F, Lang FF, McCutcheon IE, Hassenbusch SJ, Holland E: A multivariate analysis of 416 patients with glioblastoma multiforme: Prognosis, extent of resection, and survival. J Neurosurg 95: 190-198, 2001

24. Lampson LA: Molecular bases of the immune response to neural antigens. Trends Neurosci 10: 211-216, 1987

25. Linn F, Seo K, Hossmann KA: Experimental transplantation gliomas in the adult cat brain. Acta Neurochir 99: 85-93, 1989

26. Miura FK, Alves MJF, Rocha MC, Silva RD, Oba-Shinjo SM, Marie SKN: Xenograft transplantation of human malignant astrocytoma cells into immunodeficient rats: An experimental model of glioblastoma. Clinics 65: 305-309, 2010

27. Mourad PD, Farrell L, Stamps LD, Chicoine MR, Silbergeld DL: Why are systemic glioblastoma metastases rare? Systemic and cerebral growth of mouse glioblastoma. Surg Neurol 63: $511-519,2005$
28. Parsa AT, Chakrabarti I, Hurley PT, Chi JH, Hall JS, Kaiser MG, Bruce JN: Limitations of the C6/Wistar rat intracerebral glioma model: Implications for evaluating immunotherapy. Neurosurgery 47: 993-1000, 2000

29. Pirko I, Fricke ST, Johnson AJ, Rodriguez M, Macura SI: Magnetic resonance imaging, microscopy, and spectroscopy of the central nervous system in experimental animals. NeuroRx 2:250-264, 2005

30. Plate K, Breier G, Millauer B, Ullrich A, Risau W: Up-regulation of vascular endothelial growth factor and its cognate receptors in a rat glioma model of tumor angiogenesis. Cancer Res 53: 5822-5827, 1993

31. Proescholdt MA, Merrill MJ, Ikejiri B, Walbridge S, Akbasak A, Jacobson S, Oldfield EH: Site-specific immune response to implanted gliomas. J Neurosurg 95: 1012-1019, 2001

32. Reavey-Cantwell JF, Haroun RI, Zahurak M, Clatterbuck RE, Parker RJ, Mehta R, Fruehauf JP, Brem H: The prognostic value of tumor markers in patients with glioblastoma multiforme: Analysis of 32 patients and review of the literature. J Neurooncol 55: 195-204, 2001

33. Shu Q, Wong KK, Su JM, Adesina AM, Yu LT, Tsang $Y$, Antalffy BC, Baxter P, Perlaky L, Yang J: Direct orthotopic transplantation of fresh surgical specimen preserves CD133+ tumor cells in clinically relevant mouse models of medulloblastoma and glioma. Stem Cells 26:1414-1424, 2008

34. Solinas G, Germano G, Mantovani A, Allavena P: Tumorassociated macrophages (TAM) as major players of the cancer-related inflammation. J Leukoc Biol 86:1065-1073, 2009

35. Strojnik T, Kavalar R, LAH TT: Experimental model and immunohistochemical analyses of U87 human glioblastoma cell xenografts in immunosuppressed rat brains. Anticancer Res 26: 2887-2900, 2006

36. Tator $\mathrm{CH}$, Day A, $\mathrm{Ng} \mathrm{R}$, Liberman L: Chemotherapy of an experimental glioma with nitrosoureas. Cancer Res 37:476481, 1977

37. Vince $\mathrm{GH}$, Bendszus $\mathrm{M}$, Schweitzer $\mathrm{T}$, Goldbrunner $\mathrm{RH}$, Hildebrandt S, Tilgner J, Klein R, Solymosi L, Tonn JC, Roosen $\mathrm{K}$ : Spontaneous regression of experimental gliomas-an immunohistochemical and MRI study of the C6 glioma spheroid implantation model. Exp Neurol 190: 478-485, 2004

38. Weidner N, Gasparini G: Determination of epidermal growth factor receptor provides additional prognostic information to measuring tumor angiogenesis in breast carcinoma patients. Breast Cancer Res Treat 29: 97-107, 1994

39. Yusubalieva G, Baklaushev V, Gurina O, Tsitrin E, Chekhonin $\mathrm{V}$ : Immunochemical analysis of glial fibrillary acidic protein as a tool to assess astroglial reaction in experimental C6 glioma. Bull Exp Biol Med 149: 125-130, 2010 